\title{
DELTA SCUTI STARS: THEORY
}

\author{
J. A. GUZIK
}

Los Alamos National Laboratory

XTA, MS B220, Los Alamos, NM 87545

E-mail: joy@lanl.gov

\section{Introduction}

The purpose of asteroseismology is not only to derive the internal structure of individual stars from their observed oscillation frequencies, but also to test and extend our understanding of the physics of matter under the extremes of temperature, density, and pressure found in stellar interiors. In this review, I hope to point out what we can learn about the Sun by studying $\delta$ Scuti stars, as well as what we can learn about stars more massive or evolved than the Sun. I will discuss some of the difficulties in theoretical approaches to asteroseismology for $\delta$ Scuti stars, using FG Vir, $\delta$ Scuti, and CD $-24^{\circ} 7599$ as examples.

\section{Why Study $\delta$ Scuti Stars?}

The study of $\delta$ Scuti stars can provide a bridge in our knowledge between the Sun and more massive or evolved stars. We have enough confidence in our modeling and input physics from the reasonably good agreement between solar models and helioseismic inversions that we can now apply these modeling tools to stars somewhat more massive than the Sun. $\delta$ Scuti stars are main sequence or slightly post-main sequence (luminosity class III-V) stars of $1.4-3 \mathrm{M}_{\odot} . \delta$ Scuti stars are burning hydrogen either in a convective core, or in a shell outside a H-depleted core, predominantly by CNO-cycle processing, rather than the pp-chains of the Sun. The Sun has a convective envelope extending through the outer $30 \%$ of its radius, and no convective core. Main sequence $\delta$ Scuti stars have convective cores that comprise $\sim 20 \%$ of the inner radius, and very thin convection sones in the $H$ and $H e$ ionization regions near the surface. The Sun's pulsations are stochastically excited by convection, and have radial velocity amplitudes of meters/sec, whereas $\delta$ Scuti pulsations are driven by the $\kappa$ effect $(H$ and He ionization valving), and have amplitudes up to tens of $\mathrm{km} / \mathrm{sec}$. The Sun's surface rotation velocity is $\sim 2 \mathrm{~km} / \mathrm{sec}$, and its internal rotation profile has been determined rather well from helioseismic inversions. The measured $v$ sin $i$ values of $\delta$ Scuti stars range from 10 to over $200 \mathrm{~km} / \mathrm{sec}$ (Solano and Fernley 1997). It may be possible to measure portions of the internal rotation profile using oscillation frequencies (Goupil et al. 1996). 


\section{Constraints for Seismology}

We have measured most properties of the Sun much more accurately than we can for other stars. We know its mass, luminosity, age, and surface abundance ratio $\mathrm{Z} / \mathrm{X}$ quite accurately. For $\delta$ Scuti stars, we can determine luminosities if we know their distance (the Hipparcos parallax measurements will help for some $\delta$ Scuti stars). We can determine the effective temperature, surface gravity, and composition from photometric colors and spectra. For $\delta$ Scuti stars in clusters, we can better estimate the age, luminosity, and abundances by deriving these properties for the cluster as a whole (see, e.g., Hernandez et al. 1998 (Praesepe); Audard et al. 1996 (Hyades)).

Since the photospheres of both the Sun and $\delta$ Scuti stars are too cool to exhibit He lines in the visible, we cannot measure the photospheric He abundance directly. For the Sun, we now have strong evidence that diffusive settling has decreased the abundance of He and possibly of heavier elements in the envelope convection zone. The photospheric abundances of $\delta$ Scuti stars show wide variety and anomalies (Russell 1995). We have yet to determine how these abundances may have been affected by diffusive settling, radiative levitation, or mass loss.

Since we can resolve the disk of the Sun, we have been able to observe millions of $p$-modes of degrees $\ell=0-1000$. For $\delta$ Scuti stars, since we cannot resolve the disk, we can only detect by photometry the low-degree modes $(\ell=0-2$, and perhaps 3$)$ with intensities or radial velocities that do not average out over the disk. By examining variations in spectral line profiles of rapidly rotating $\delta$ Scuti stars, a number of researchers (e.g. Kennelly and Walker 1996) have been able to detect some higher-degree nonradial modes. We can expect to observe dozens of predicted unstable radial and nonradial modes, pure $p$-modes, modes with a mixed $p$ - and $g$-mode character, and possibly pure $g$-modes. The modes with $g$-type nodes are potentially very useful in probing the convective core size and measuring the distance of convective overshooting (Dziembowski and Pamyatnykh 1991; Audard et al. 1995).

Mode identification for solar p-modes is straightforward, since we can detect all of the expected modes, and measure the frequencies very accurately. But mode identification is problematic for $\delta$ Scuti stars. Not all of the predicted modes are observed, and it is difficult to find a pattern that gives clues to the mode identification. Progress has been made recently in mode identification for a few $\delta$ Scuti stars (e.g., 4 CVn and FG Vir) using spectroscopic methods (Breger et al., these proceedings; Viskum et al. 1998), but the identification of some modes remains ambiguous.

\section{Approaching Asteroseismology}

An example of a star that is promising for asteroseismology is the $\delta$ Scuti star FG Vir (Breger et al. 1995, 1997). FG Vir is observed to have $\mathrm{T}_{\text {eff }}=7500 \pm 150 \mathrm{~K}$ and $\log g \sim 4.0$. The estimated rotation velocity is $\sim 42 \mathrm{~km} / \mathrm{sec}$ (Mantegazza et al. 1994). Breger et al. (1997) report 21 observed frequencies for FG Vir, including some that likely have mixed $p$ - and $g$-type character. The luminosity derived from the Hipparcos parallax, in addition to spectroscopic mode identification, indicate that the sixth highest amplitude mode at $140.7 \mu \mathrm{Hz}$ is probably the radial fundamental mode. Several other modes have been identified by spectroscopic methods.

It is difficult to determine the intrinsic metallicity of FG Vir. The measured photospheric $[\mathrm{Fe} / \mathrm{H}]$ is 0.65 dex, much higher than solar, whereas $[\mathrm{C} / \mathrm{H}]=-0.63$, much lower than solar. Such anomalies are expected due to diffusive settling and radiative levitation of elements (Smith 1996). The abundances of other elements less affected 
by settling or levitation, such as $[\mathrm{Ca} / \mathrm{H}]$ and $[\mathrm{Ti} / \mathrm{H}]$, are respectively 0.24 and 0.19 dex, which indicates that FG Vir may have an intrinsic $Z$ of about 0.03 .

Our goal is to find a model that matches all of these observational constraints for FG Vir. However, we also need to consider the uniqueness of the model. There are many areas of parameter space to explore, such as the helium (Y) or heavy element abundance $(Z)$, element mixture, and internal rotation profile, which may make it difficult to find a unique model. We may be able to use asteroseismology to determine the $\mathrm{Y}$ abundance (Monteiro et al., these proceedings), the $\mathrm{Z}$ abundance (Gusik et al. 1998), the internal rotation profile (Goupil et al. 1996), or indicate the need for improved opacities (Brown et al. 1994), or convective overshoot (Monteiro et al., these proceedings; Dziembowski and Pamyatnykh 1991; Audard and Roxburgh 1997). Applying our asteroseismological tools to many $\delta$ Scuti stars will be necessary to distinguish between solutions that may produce the same effect. For example, can we discriminate between the effects of increased $\mathrm{Z}$ or convective core overshoot, both of which enlarge the convective core?

Here we examine the effect of metallicity on the frequencies of candidate FG Vir models. Guzik et al. (1998) identified two models in their evolution and pulsation calculations of mass 1.82 and $1.95 \mathrm{M}_{\odot}$, with $\mathrm{Z}=0.02$ and 0.03 , respectively, that match many of the observational constraints of FG Vir ( $T_{\text {eff }}, \log g$, radial fundamental mode at $140.7 \mu \mathrm{Hz}$ ). These models do not include convective overshoot. Table 1 compares the model properties. Without additional pulsation frequencies, these models would be indistinguishable from each other observationally, except perhaps for their photospheric abundances (which may not be representative of the interior abundance due to diffusion/levitation). However, the interior structures of these models, particularly the convective core size and degree of core H-depletion, are significantly different.

TABLE 1. FG Vir Model Properties

\begin{tabular}{lll}
\hline Model Property & $1.82 \mathrm{M}_{\odot}$ & $1.95 \mathrm{M}_{\odot}$ \\
\hline $\mathrm{Z}$ & 0.02 & 0.03 \\
$\mathrm{R}\left(\mathrm{R}_{\odot}\right)$ & 2.26 & 2.31 \\
$\mathrm{M} / \mathrm{R}^{3}$ & 0.1575 & 0.1580 \\
$\mathrm{~T}_{\text {.ff }}(\mathrm{K})$ & 7368 & 7412 \\
$\log \mathrm{g}$ & 3.99 & 4.00 \\
$\log \mathrm{L} / \mathrm{L}_{\odot}$ & 1.13 & 1.16 \\
$\mathrm{X}_{\text {conveore }}$ & 0.257 & 0.355 \\
$\mathrm{R}_{\text {convcore }}\left(\mathrm{R}_{\odot}\right)$ & 0.175 & 0.220 \\
$\mathrm{M}_{\text {convcore }}\left(\mathrm{M}_{\odot}\right)$ & 0.155 & 0.181 \\
Age $(\mathrm{Gyr})$ & 0.879 & 0.731 \\
\hline
\end{tabular}

Figure 1 shows the calculated radial $(\ell=0)$ and nonradial $(\ell=1$ and 2 ) modes for the two models plotted against each other, assuming for now no rotational splitting $(m=0)$. If all of the calculated frequencies were identical, the points would lie on a straight line. One can see that the predicted radial mode frequencies are nearly identical, which is not surprising since these models have nearly the same mean density. For the nonradial modes, there are a few interesting differences. For $\ell=1$, there is 


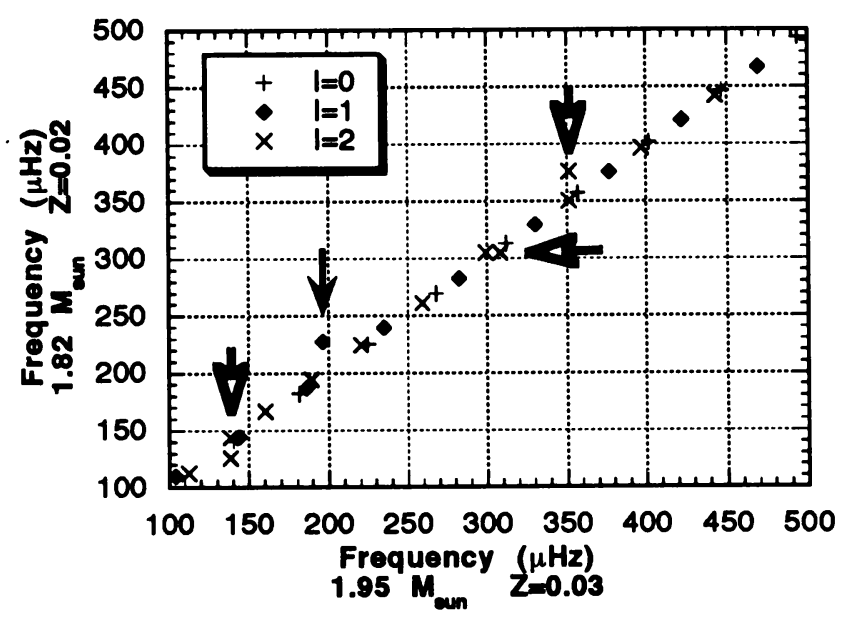

Figure 1. Calculated frequencies of the two candidate FG Vir models from Table 1 plotted against each other. A few nonradial modes are shifted (solid arrow) in frequency or added (open arrows) due to the differences in internal structure between models.

a $30 \mu \mathrm{Hz}$ shift between models in the mode near $200 \mu \mathrm{Hz}$, as a consequence of the difference in convective core size. For $\ell=2$, the more evolved $1.82-\mathrm{M}_{\odot}$ model has two closely-spaced frequencies near $140 \mu \mathrm{Hz}$, and again at $350 \mu \mathrm{Hz}$, whereas the 1.95$\mathrm{M}_{\odot}$ model has only one mode near these frequencies. The $1.95-\mathrm{M}_{\odot}$ model has two closely-spaced modes near $300 \mu \mathrm{Hz}$, whereas only one mode exists near this frequency for the $1.82-\mathrm{M}_{\odot}$ model. It is important to note that the modes with nearly identical frequencies in each model may have one more or less radial node, or a p-type instead of a $g$-type node, and therefore significantly different eigenfunctions.

How well do these models match the frequencies reported for FG Vir? Taking into account plausible first-order rotational splitting for the nonradial modes, and adopting the additional spectroscopic mode identifications of Breger et al. (these proceedings) and Viskum et al. (1998), neither model simultaneously matches all 21 frequencies (see Guzik et al. 1998). The fit would be improved if we could find a model in which a mode is added (compared to the $1.95-\mathrm{M}_{\odot}$ model) or shifted (compared to the 1.82$\mathrm{M}_{\odot}$ model) near $130 \mu \mathrm{Hz}$, and also in which the $\ell=2$ mode near $260 \mu \mathrm{Hz}$ is shifted to $\sim 250 \mu \mathrm{Hz}$. These clues can be used in the next iteration to evolve models that more closely match the observed frequencies.

\section{Prospects for Asteroseismology of More-Evolved $\delta$ Scuti Stars}

It may prove difficult to do asteroseismology on the more evolved $\delta$ Scuti stars that are burning hydrogen in a shell outside the $\mathrm{H}$-depleted core, due to the very dense spectrum of predicted modes (mainly high-order $g$-modes) that have not yet been detected, and the difficulty in mode identification given these predictions. As an example, we consider $\delta$ Scuti itself. Templeton et al. (1997) reported six frequencies in the range 54-99 $\mu \mathrm{Hz}$ for this star. Templeton et al. constructed evolution and pulsation models with masses 2.1 and $2.4 \mathrm{M}_{\odot}$, and $\mathrm{Z}=0.02$ and $\mathrm{Z}=0.06$, respectively, that match the observed spectral type, luminosity (given the Hipparcos parallax), and identified 
radial fundamental mode frequency $(57.731 \mu \mathrm{Hz})$ of $\delta$ Scuti. For the $2.4-\mathrm{M}_{\odot}, \mathrm{Z}=0.06$ model with the larger mode spacing, there are 3 unstable radial modes, 13 unstable $\ell=1$ modes, and 19 unstable $\ell=2$ modes. Accounting for rotational splitting of the nonradial modes into $2 \ell+1$ frequencies, this model has 137 possible modes! $A$ range of $\ell=2$ modes, and often at least one $\ell=1$ mode can match the five remaining observed frequencies. It is possible that other predicted modes have a very low amplitude and will eventually be detected, but we may need to determine instead why only a few modes (and which modes) are excited to observable amplitudes. Driembowski and Krolikowska (1990) propose that the $\ell=1$ modes may be preferentially selected due to partial mode trapping. For $\delta$ Scuti, this would narrow the choice of frequency matches to only one or two $\ell=1$ modes per observed frequency.

As mentioned by Breger (these proceedings), there may be observational indications for the evolved $\delta$ Scuti star $4 \mathrm{CVn}$ that this dense spectrum of predicted $g$-modes does not occur at all, as though the $g$-type nodes have been somehow suppressed in the core. Breger finds that the pattern of observed modes in $4 \mathrm{CVn}$ is consistent with a sequence of radial modes and rotationally-split nonradial p-modes. If such patterns are found in the frequencies of other evolved $\delta$ Scuti stars, explaining these observations will pose an interesting challenge to the theoreticians!

\section{The Importance of Rotation}

To match observed frequencies in detail, and also to derive the internal rotation profile for a $\delta$ Scuti star, it is critical to take into account the rotational splitting of modes. To date, rotational effects have been taken into account mainly using perturbation theory approaches. The angular eigenfunctions of nonradial modes are described by spherical harmonics $\left(Y_{\ell}^{m}\right)$, in which $\ell$ is the number of node lines on the surface, and $m$ the number of node lines through the rotation axis. To first order, rotation breaks the $m$ frequency degeneracy, by accounting for the Coriolis force, which is proportional to $\Omega \times v$. When $\Omega$ is a function of radius only (i.e. the rotation rate has no latitudinal dependence), the nonradial modes are split into $2 \ell+1$ equally-spaced prograde and retrograde modes. First-order corrections neglect the distortion of the star from a sphere, so the radial mode frequencies are not affected.

For moderately-rotating $\delta$ Scuti stars, second-order corrections become significant. Second-order corrections account for the centrifugal force $(\Omega \times \Omega \times r)$, and include distortion of the star from a sphere. These corrections shift radial modes as well as nonradial, and destroy the symmetrical $m$-splitting of nonradial modes. Second-order corrections are derived under various approximations by Saio (1981; solid body rotation, include overall shift in multiplet frequencies), Gough and Thompson (1990; include magnetic fields, and overall shift in average multiplet frequency due to distortion, but neglect latitudinal dependence of rotation), and Dziembowski and Goode (1992; include radial and latitudinal dependence of $\Omega$, but neglect overall shift in multiplet frequencies). Accidental degeneracies between modes of different degree $\ell$ also affect mode frequencies, and must be taken into account (Soufi et al. 1998).

Figure 2 illustrates the first- and second-order rotational splitting corrections for a model of CD $-24^{\circ} 7599$ (Bradley and Guzik 1997), with assumed uniform rotation velocity of $75 \mathrm{~km} / \mathrm{sec}$ (Handler et al. 1997). The solid horizontal lines indicate the 13 observed frequencies of Handler et al. Second-order corrections are estimated using the formula of Saio (1981), strictly valid for $n=3$ polytropes and uniform rotation. As can be seen, the first-order corrections split the modes evenly, and are $\sim 10 \mu \mathrm{Hz}$ 


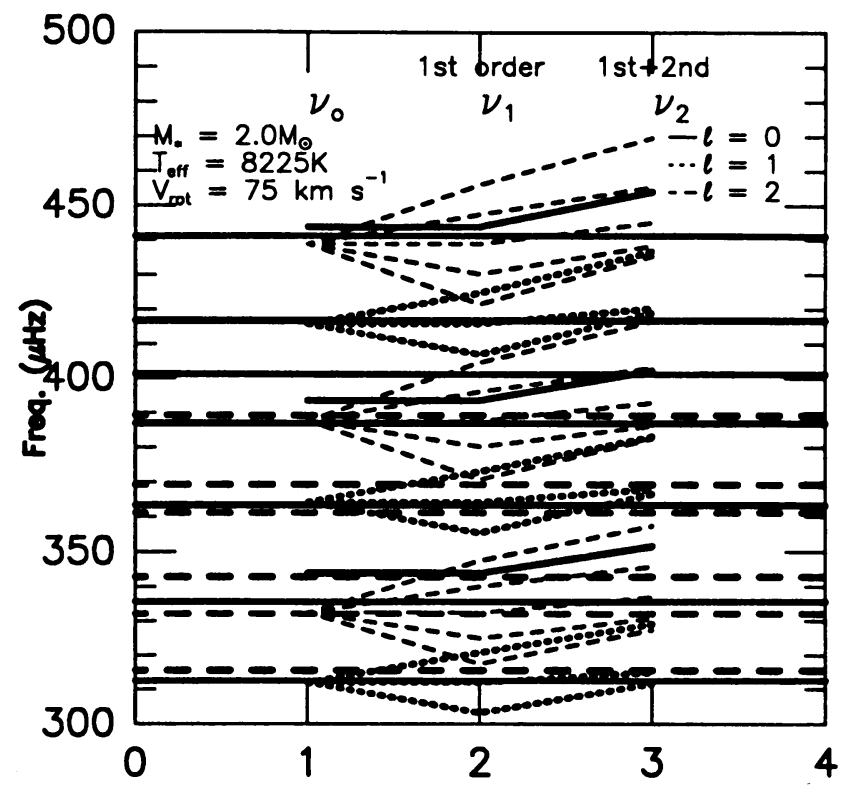

Figure 2. A schematic illustration of the importance of rotational splitting on the frequencies of CD $-24^{\circ}$ 7599. $\nu_{0}$ are frequencies without rotational splitting, $\nu_{1}$ includes first order rotation corrections, and $\nu_{2}$ includes both first and second-order corrections.

per $\Delta m$. The second-order corrections shift all of the multiplets to higher frequency, and break the even spacing within a multiplet. The radial modes are shifted by $\sim 10$ $\mu \mathrm{Hz}$. Considering the predicted spectrum of frequencies, with modes of different $\ell$ values now lying very close to each other, it will be important to include the effects of mode coupling. It will also be very difficult to disentangle mode identifications without additional information.

Third-order corrections are also significant for even moderately rotating $\delta$ Scuti stars. Soufi et al. (1998) are working out a third order perturbation theory formalism. Ultimately perturbation theory may be inadequate for the most rapidly rotating stars $(v \sim 200 \mathrm{~km} / \mathrm{sec})$ and we may need to calculate the evolution and pulsations of two- or three-dimensional differentially rotating distorted models. There has been progress in this direction, e.g. the two-dimensional evolution/hydrodynamics modeling of Deupree $(1995,1997)$, and the three-dimensional pulsation modeling of Clement (1993).

\section{What Can We Learn about the Sun from $\delta$ Scuti Stars?}

Since $\delta$ Scuti stars cover a wide range of parameter space in mass, composition, temperature, rotation rate, etc., there are a number of areas where improvements in the physics of modeling $\delta$ Scuti stars will feed back into solar modeling. First, we may be able to verify or improve microphysics (opacities, equation of state, diffusion treatments, nuclear reaction rates). Brown et al. (1994), applying inversion techniques to hypothetical frequency spectra of sun-like stars, find that oscillation frequencies can be used to distinguish among models using different opacities or equations of state. 
The photospheric composition and pulsation driving of $\delta$ Scuti stars are enigmatic. Diffusion time-scales in the stellar envelope are much shorter for the $\delta$ Scuti stars than for the Sun due to their very small envelope convection zones. The amount of helium diffused from the pulsation driving layers directly affects the pulsational stability (Cox et al. 1979), amplitudes (Guzik 1993), and light curve shapes (Guzik 1992). Helium settling and selective levitation of some elements may be mitigated by mass loss; a not-unreasoanble mass loss rate of $10^{-12} \mathrm{M}_{\odot} / \mathrm{yr}$ would be required to "keep up" with diffusion (Guzik and Cox 1991). If we can use pulsation properties to learn more about abundances in the pulsation driving region of $\delta$ Scuti stars, we may be able to better test or calibrate calculations and theories of diffusion, mixing, or mass loss proposed for the Sun to explain the solar $\mathrm{Li}$ abundance, or the discrepancy between inferred and calculated sound speed at the convection zone base for standard solar models including helium and element diffusion.

Surface boundary conditions are another area in which we may gain insight from $\delta$ Scuti stars. Many of the remaining discrepancies between observed and calculated solar oscillation frequencies are due to problems with solar models near the superadiabatic gradient at the top of the solar convection zone $(\sim 8,000$ to $10,000 \mathrm{~K})$. Increased low-temperature opacities, nonequilibrium radiation diffusion, nonadiabaticity, turbulent pressure, and more sophisticated convection treatments have all been proposed to alleviate this discrepancy (see Guzik et al. 1996). Li and Stix (1994) discuss moving the outer boundary of $\delta$ Scuti models to a smaller optical depth to reproduce the instability strip blue edge. Audard et al. (1998) discuss treatments of model atmospheres for $\delta$ Scuti and Ap stars that improve agreement with the acoustic cutoff frequency. Perhaps investigating problems such as these will provide support for one or more proposed improvements in solar atmosphere modeling.

There are a number of questions raised by helioseismic measurements of the solar internal rotation. How did the Sun's current rotation profile evolve? Do other stars show latitudinally-dependent rotation rates that persist throughout the envelope? Are other stars more rapidly rotating in their cores than at their photospheres? Hopefully, systematics of rotational splitting due to differential rotation will answer these questions for other stars, and help us determine whether the Sun is typical or anomalous.

Finally, there is hope to study $g$-mode driving, amplitudes, detectability, and mode selection, to learn more about what we can expect to observe for $g$-modes in the Sun.

\section{Discussion}

M. Paparo: I would like to call your attention to $\theta$ Tuc, for which ten frequencies were obtained which have extremely regular spacing (Paparo et al. 1996). This gives some guidelines for mode identification. Mode identification based on colour data is coming out soon (Sterken 1997). The modeling of $\theta$ Tuc is probably easier than the complex case of FG Vir.

J. Gusik: Yes, I am aware of this star, and my graduate student M. Templeton is trying to model it.

\section{Acknowledgements}

The author is especially grateful to P. Bradley, A. Cox, M. Templeton, M. Breger, W. Driembowski, D. Kurtz, and J. Christensen-Dalsgaard for useful discussions and 
assistance. This work was funded by a NASA Astrophysics Theory grant.

\section{References}

Audard, N., Provost, J. and Christensen-Dalsgaard, J. 1995, Astron. Astrophys. 297, 427.

Audard, N., et al.: 1996, Bull. Astron. Soc. India 24, 305.

Audard, N. and Roxburgh, I.W.: 1997, in F. Schmider and J. Provost (eds.) IA U Colloguium 181: Sounding Solar and Stellar Interiors, in press.

Audard, N., Kupka, F., Morel, P. and Weiss, W.W. Atmosphere Models of A-F Stars. Effects on the Acoustic Cut-off Frequency, 1998, Astron. Astrophys., submitted.

Bradley, P.A. and Guzik, J.A.: 1997, in F. Schmider and J. Provost (eds.) IA U Colloquium 181: Sounding Solar and Stellar Interiors, in press.

Breger, M. et al.: 1995, Astron. Astrophys. 297, 473.

Breger, M. et al.: 1997, Astron. Astrophys., in press.

Brown, T.M., Christensen-Dalsgaard, J., Weibel-Mihalas, B., and Gilliland, R.: 1994, Astrophys. J. 427, 1013.

Clement, M.J.: 1993, Astrophys. J. 406, 651.

Cox, A.N., King, D.S., and Hodson, S.W.: 1979, Astrophys. J. 231, 798.

Cox, J.P.: 1984, Publ. Astron. Soc. Pacific 96, 577.

Deupree, R.G.: 1995, Astrophys. J. 439, 357.

Deupree, R.G.: 1997, Stellar Evolution with Arbitrary Rotation Laws III. Convective Core Overshoot and Angular Momentum Distribution, Astrophys. J., in press.

Dziembowski, W.A. and Krolikowska, M.: 1990, Acta Astronomica 40, 19.

Driembowski, W.A. and Pamyatnykh, A.A.: 1991, Astron. Astrophys. 248, L11.

Dziembowski, W.A. and Goode, P.R.: 1992, Astrophys. J. 394, 670.

Gough, D.O. and Thompson, M.J.: 1990, MNRAS 242, 25.

Goupil, M.J., Dziembowski, W.A., Goode, P.R., and Michel, E.: 1996, Astron. Astrophys. $305,487$.

Guzik, J.A. and Cox, A.N.: 1991, Delta Scuti Star Newoletter, ed. M. Breger, 3, 6.

Guzik, J.A.: 1992, Delta Scuti Star Newaletter, ed. M. Breger, 5, 8.

Guzik, J.A.: 1993, in J. Nemec and J. Matthews (eds.) New Perspectives on Stellar Pulsation and Pulsating Variable Stars, Cambridge U. Press, Cambridge, p. 243.

Gurik, J.A., Cox, A.N., and Swenson, F.J.: 1996, Bull. Astron. Soc. India 24, 161.

Guzik, J.A., Bradley, P.A., and Templeton, M.R. et al.: 1998, in P. Bradley and J. Guzik (eds.) A Half Century of Stellar Pulsation Interpretations, in press.

Hernandez, M.M. et al.: 1998, in P. Bradley and J. Guzik (eds.) A Half Century of Stellar Pulsation Interpretations, in press.

Handler, G. et al.: 1997, Astron. Astrophys. 286, 303.

Kennelly, E.J. and Walker, G.A.H.: 1996, Publ. Astron. Soc. Pacific 108, 327.

Li, Y. and Stix, M.: 1994, Astron. Astrophys. 286, 815.

Mantegazza, L., Poretti, E., and Bossi, M.: 1994, Astron. Astrophys. $287,95$.

Paparo, M., Sterken, C., Spoon, H., and Birch, P.V.: 1997, Astron. Astrophys. 315, 400.

Russell, S.C.: 1995, Astrophys. J. 451, 747.

Saio, H.: 1981, Astrophys. J. 244, 299.

Smith, K.C.: 1996, Astrophys. Space Sci. 237, 77.

Solano, E. and Fernley, J.: 1997, Astron. Astrophys. Suppl. Ser. 122, 131.

Soufi, F., Goupil, M.J., and Driembowski, W.A.: 1998, Astron. Astrophys., in preparation.

Sterken, C.: 1997, Astron. Astrophys. 325, 563.

Templeton, M.R. et al.: 1997, Astron. J. 114, 1592.

Viskum, M. et al.: 1998, in P. Bradley and J. Guzik (eds.) A Half Century of Stellar Pulsation Interpretations, in press. 